\title{
Qualitative analysis of a SIR epidemic model with saturated treatment rate
}

\author{
Zhang Zhonghua $\cdot$ Suo Yaohong
}

Received: 2 February 2009 / Published online: 21 August 2009

(C) Korean Society for Computational and Applied Mathematics 2009

\begin{abstract}
On account of the effect of limited treatment resources on the control of epidemic disease, a saturated removal rate is incorporated into Hethcote's SIR epidemiological model (Hethcote, SIAM Rev. 42:599-653, 2000). Unlike the original model, the model has two endemic equilibria when $R_{0}<1$. Furthermore, under some conditions, both the disease free equilibrium and one of the two endemic equilibria are asymptotically stable, i.e., the model has bistable equilibria. Therefore, disease eradication not only depends on $R_{0}$ but also on the initial sizes of all sub-populations. By the Poincaré-Bendixson theorem, Poincaré index, center manifold theorem, Hopf bifurcation theorem and Lyapunov-Lasalle theorem, etc., the existence and asymptotical stability of the equilibria, the existence, stability and direction of Hopf bifurcation are discussed, respectively.
\end{abstract}

Keywords Poincare index $\cdot$ Bistable equilibria $\cdot$ Center manifold theorem $\cdot$ Hopf bifurcation · Stability

Mathematics Subject Classification (2000) 34D20 · 34C23 · 92C37

\section{Introduction}

Epidemic model can contribute to the design and analysis of epidemiological surveys, suggesting crucial data that should be collected, identifying trends, making general

This work was supported by the Training Fund of Xi' an University of Science and Technology under the contract 200836 and the Dr. Start-up Fund of Xi' an University of Science and Technology.

Z. Zhonghua $(\bowtie) \cdot S$. Yaohong

School of Sciences, Xi' an University of Science and Technology, Xi' an 710054, China

e-mail: wwwzhonghua@sohu.com

S. Yaohong

School of Aerospace, Xi' an Jiaotong University, Xi'an 710049, China 
forecasts, and estimating the uncertainty in forecasts [6]. Therefore, to add new factors such as different treatment plans, etc., to classical epidemic model and then investigate their effects on the dynamical behaviors has become a focus in applied mathematics. In [2, 3, 7, 10, 15, 17] and references therein, several kinds of removal rates, i.e., the recovery from the infected subpopulation per unit time, have been adopted to characterize different treatment truths of epidemic disease. For example, in [15], W. Wang and S. Ruan suppose a removal rate has the form

$$
h(I)= \begin{cases}r, & I>0, \\ 0, & I=0,\end{cases}
$$

and formulate the following model

$$
\left\{\begin{array}{l}
\frac{d S}{d t}=A-d S-\lambda S I, \\
\frac{d I}{d t}=\lambda S I-(d+\gamma) I-h(I), \\
\frac{d R}{d t}=\gamma I+h(I)-d R,
\end{array}\right.
$$

where $S, I$, and $R$ denote the numbers of the susceptible, infective and recovered individuals at time $t$, respectively. Then they discuss the stability of equilibria and prove the model exhibits Bogdanov-Takens bifurcation, Hopf bifurcation and Homoclinic bifurcation. Obviously, the removal rate of (1) is a reasonable approximation to the treatment truth of the disease that human possesses rich treatment sources and better treatment techniques. However, for the new outbreak disease such as SARS, etc., maybe saturated treatment rate is a better alternative. In fact, the treatment rate is small for there is short of effective treatment techniques at the beginning of the outbreak. Then, the treatment rate will be increased with the improving of the hospital's treatment conditions including effective medicines, skillful techniques, etc. At last, for the treatment capacity of any community is limited, the treatment will reach to its maximum if the number of infective individuals is large enough. In view of above description, we suppose the removal rate to be

$$
h(I)=\frac{\beta I}{1+\alpha I}, \quad I \geq 0, \alpha, \beta>0 .
$$

Clearly, the epidemicity of disease is closely related to the stability of the equilibria of mathematical models. In classical models there usually exist a disease-free equilibrium and a unique endemic equilibrium. The stability of the disease free equilibrium is determined by a threshold parameter $R_{0}$, known as the basic reproductive number, i.e. the disease free equilibrium is asymptotically stable if $R_{0}<1$, while it is unstable and the unique endemic equilibrium is asymptotically stable if $R_{0}>1$. However, recent studies have showed that some epidemiological models (see [1, 9, $12,14]$ for examples) have multiple endemic equilibria, and which can exist simultaneously even $R_{0}$ is less than the unity. Furthermore, the disease free equilibrium and one of the endemic equilibria can be stable simultaneity, i.e. there exist bistable equilibria. Therefore, it is not always effective to eliminate the disease by reducing $R_{0}$ to the values less than 1 . In fact, to eradicate this kind of disease, one not only 
needs to reduce $R_{0}$ but also needs to restrict the initial value of each subpopulation to the domain of attraction of the disease free equilibrium.

In the present paper, considering the saturated removed rate and supposing the removal individuals perpetual immunity, a new SIR epidemic model is formulated. Then, we discuss the existence and asymptotical stability of equilibria and the Hopf bifurcation. It is interesting that out model has bistable equilibria in two cases: $R_{0}<$ 1 and $\Delta=0$ (will be defined in the text); $R_{0}<1$ and $\Delta>0$. The remainder of this paper is arranged as follows: Sect. 2 presents the existence of equilibria, Sect. 3 researches the stability of every equilibrium, the existence, stability and direction of Hopf bifurcation, Sect. 4 is the numerical simulation which verifies our qualitative results, Sect. 5 makes the conclusions.

\section{The existence of equilibria}

Obviously, incorporating the saturated treatment rate (3) into model (2) leads to

$$
\left\{\begin{array}{l}
\frac{d S}{d t}=A-d S-\lambda S I, \\
\frac{d I}{d t}=\lambda S I-(d+\gamma) I-\frac{\beta I}{1+\alpha I}, \\
\frac{d R}{d t}=\gamma I+\frac{\beta I}{1+\alpha I}-d R,
\end{array}\right.
$$

where $A$ is the recruitment rate of the population, $d$ the natural death rate of the population, $\gamma$ the natural recovery rate of the infective individuals, $\lambda S I$ the bilinear incidence rate and $\frac{\beta I}{1+\alpha I}$ the removal rate of the infected individuals.

Remark 1 It is easy to see that the treatment rate $h(I)=\frac{\beta I}{1+\alpha I}$ is a continuously differentiable function of $I$ and $: h(0)=0, \frac{d h(I)}{d I}>0, \lim _{I \rightarrow \infty} h(I)=\frac{\beta}{\alpha}$, where $\frac{\beta}{\alpha}$ is the maximal treatment capacity of some a community.

Noticing the last equation of (4) is independent of the others, we mainly investigate the dynamic properties of $S$ and $I$ in the present paper. Therefore, only the following subsystem is focused on.

$$
\left\{\begin{array}{l}
\frac{d S}{d t}=A-d S-\lambda S I, \\
\frac{d I}{d t}=\lambda S I-(d+\gamma) I-\frac{\beta I}{1+\alpha I} .
\end{array}\right.
$$

For system (5), we have

Lemma $2 \mathbb{O}=\left\{(S, I) \mid S \geq 0, I \geq 0, S+I \leq \frac{A}{d}\right\}$ is a positively invariant region of system (5).

By the Bendixson-Dulac criterion and Lemma 1, we have

Lemma 3 If $\lambda<\frac{d^{3}}{A^{2} \alpha}$, system (5) does not have closed orbit in the first quadrant of $(S, I)$ plane. 
Proof Let $D=\frac{1+\alpha I}{S I}, P=A-d S-\lambda S I$ and $Q=\lambda S I-(d+\gamma) I-\frac{\beta I}{1+\alpha I}$. After computation, we get

$$
\frac{\partial D P}{\partial S}+\frac{\partial D Q}{\partial I}=\frac{\lambda \alpha S^{2} I-A \alpha I-n \alpha S I-A}{S^{2} I} \leq \frac{\lambda \alpha S^{2}-A \alpha-d}{S^{2} I} .
$$

Because $\mathbb{O}$ is a positively invariant set of system (5), we restrict $(S, I)$ to the inner of $\mathbb{O}$. Therefore, we have

$$
\frac{\partial D P}{\partial S}+\frac{\partial D Q}{\partial I} \leq \frac{\lambda \alpha\left(\frac{A}{d}\right)^{3}-A}{S^{2} I} .
$$

Then, by Bendixson-Dulac criterion, system(5) does not have closed orbit in the first quadrant of $(S, I)$ plane if $\lambda<\frac{d^{3}}{A^{2} \alpha}$.

In the following, we discuss the existence of equilibria. For convenience, we introduce two notations: $R_{0}=\frac{\lambda A}{d(\beta+n)}, R_{1}=\frac{\lambda A \alpha}{n d \alpha+\lambda(\beta+n)}$, where $n=d+\gamma$ and $R_{0}$ is called the basic reproduction number in [6].

Let the right hand side of system (5) be zero. It isn't difficult to obtain that system (5) always has a disease free equilibrium $E_{0}=\left(\frac{A}{d}, 0\right)$. Then, under the condition of $I \neq 0$, solving algebraic equations by the fixed theorem yields.

\section{Theorem 4}

(A) If $R_{0}>1$, there exists a unique endemic equilibrium $E_{1}=\left(S_{1}^{*}, I_{1}^{*}\right)$.

$\left(B_{1}\right)$ If $R_{0}=1$ and $R_{1}>1$, there exists a unique endemic equilibrium $E_{2}=\left(S_{2}^{*}, I_{2}^{*}\right)$.

$\left(B_{2}\right)$ If $R_{0}=1$ and $R_{1} \leq 1$, no endemic equilibrium exists.

( $\left.C_{1}\right) R_{0}<1, R_{1}>1$ and $\Delta>0$, there exist two endemic equilibria: $E_{1}, E_{3}=$ $\left(S_{3}^{*}, I_{3}^{*}\right)$.

$\left(C_{2}\right)$ If $R_{0}<1, R_{1}>1$ and $\Delta=0$, there exists a unique endemic equilibrium $E_{4}=$ $\left(S_{4}^{*}, I_{4}^{*}\right)$.

( $\left.C_{3}\right)$ If $R_{0}<1, R_{1}>1$ and $\Delta<0$, no endemic equilibrium exists.

( $\left.C_{4}\right)$ If $R_{0}<1$ and $R_{1} \leq 1$, no endemic equilibrium exists, where $I_{1}^{*}=\frac{\lambda A \alpha-n d \alpha-(\beta+n) \lambda+\sqrt{\Delta}}{2 n \lambda \alpha}, I_{2}^{*}=\frac{\lambda A \alpha-n d \alpha-(\beta+n) \lambda}{n \lambda \alpha}, I_{3}^{*}=\frac{\lambda A \alpha-n d \alpha-(\beta+n) \lambda-\sqrt{\Delta}}{2 n \lambda \alpha}$, $I_{4}^{*}=\frac{\lambda A \alpha-n d \alpha-(\beta+n) \lambda}{2 n \lambda \alpha}, S_{j}^{*}=\frac{A}{d+\lambda I_{j}^{*}}, \Delta=(-\lambda A \alpha+n d \alpha+(\beta+n) \lambda)^{2}+$ $4 n \lambda \alpha(\lambda A-(\beta+n) d), j=1,2,3,4$.

\section{The qualitative results}

For the epidemicity of disease is closely related to the stability of the equilibria of mathematical models, we first investigate the stability of the disease free equilibrium $E_{0}$. The Jacobian matrix of system (5) at $E_{0}$ is

$$
M_{E_{0}}:=\left(\begin{array}{cc}
-d & -\frac{\lambda A}{d} \\
0 & (\beta+n)\left(R_{0}-1\right)
\end{array}\right) .
$$

Then, by the Hurwitz criterion (see $[8,11,18]$ for the details), we have 
Theorem 5 If $R_{0}>1$, the disease free equilibrium $E_{0}$ is unstable, while if $R_{0}<1$ the disease free equilibrium $E_{0}$ is asymptotically stable

Obviously, the matrix $M_{E_{0}}$ has two real eigenvalues: $0,-d$ when $R_{0}=1$, i.e. $E_{0}$ a non-hyperbolic equilibrium. We study its stability by the center manifold theorem (see $[4,16]$ for the details). Let $y_{1}=S-\frac{A}{d}, y_{2}=I$, then we have

$$
\left\{\begin{array}{l}
\frac{d y_{1}}{d t}=-d y_{1}-\frac{\lambda A}{d} y_{2}-\lambda y_{1} y_{2}, \\
\frac{d y_{2}}{d t}=\lambda y_{1} y_{2}-\frac{\alpha \beta y_{2}^{2}}{1+\alpha y_{2}} .
\end{array}\right.
$$

By the following transformation:

$$
\left(\begin{array}{l}
y_{1} \\
y_{2}
\end{array}\right)=\left(\begin{array}{cc}
1 & -1 \\
0 & \frac{d}{\beta+n}
\end{array}\right)\left(\begin{array}{l}
x_{1} \\
x_{2}
\end{array}\right)
$$

system (6) can be transformed into the standard form

$$
\left\{\begin{array}{l}
\frac{d x_{1}}{d t}=f_{1}\left(x_{1}, x_{2}\right), \\
\frac{d x_{2}}{d t}=f_{2}\left(x_{1}, x_{2}\right),
\end{array}\right.
$$

where

$$
\begin{aligned}
& f_{1}\left(x_{1}, x_{2}\right)=-d x_{1}-\frac{\lambda(\beta+n)}{d} x_{1} x_{2}-\frac{\lambda(\beta+n)^{2}}{d^{2}} x_{2}^{2}, \\
& f_{2}\left(x_{1}, x_{2}\right)=\frac{\lambda(\beta+n)}{d} x_{1} x_{2}+\frac{(\beta+n)^{2}(\lambda-\alpha \beta)}{d^{2}} x_{2}^{2}+\frac{\alpha^{2} \beta(\beta+n)^{3}}{d^{3}\left(1+\frac{\alpha(\beta+n)}{d} x_{2}\right)} x_{2}^{3} .
\end{aligned}
$$

By the center manifold theory, there exists a center manifold for system (7), which can be expressed locally by

$$
W^{c}(0)=\left\{\left(x_{1}, x_{2}\right) \in \mathbb{R}^{2}\left|x_{1}=H\left(x_{2}\right),\left\|x_{2}\right\|<\delta, H(0)=0, D H(0)=0,\right| \delta>0\right\}
$$

where $\delta$ is sufficiently small and $D H$ is the derivative of $H$ with respect to $x_{2}$.

To compute the center manifold $W^{c}(0)$, we assume $H\left(x_{2}\right)$ has the form

$$
x_{1}=H\left(x_{2}\right)=h_{2} x_{2}^{2}+h_{3} x_{2}^{3}+h_{4} x_{2}^{4}+h_{5} x_{2}^{5}+h_{6} x_{2}^{6}+\cdots .
$$

By the invariance of $W^{c}(0)$ under the dynamics of (7) yields

$$
D H \cdot f_{2}\left(x_{2}, H\left(x_{2}\right)\right)+d H\left(x_{2}\right)-f_{1}\left(x_{2}, H\left(x_{2}\right)\right)=0 .
$$

Substituting (7) into (9), and then equating coefficients on each power of $x_{2}$ to zero yields

$$
h_{2}=\frac{-\lambda k^{2}}{d}, \quad h_{3}=\frac{\lambda k^{3}}{d^{2}}(-2 k \alpha \beta+2 \lambda k+\lambda),
$$




$$
\begin{aligned}
h_{4}= & \frac{-k^{4} \lambda}{d^{3}}\left(\left(7 k+1+6 k^{2}\right) \lambda^{2}-k \alpha \beta(5+12 k) \lambda+2 k \alpha^{2} \beta(3 k \beta-d)\right), \\
h_{5}= & \frac{\lambda k^{5}}{d^{4}}\left(\left(24 k^{3}+44 k^{2}+16 k+1\right) \lambda^{3}-k \alpha \beta\left(72 k^{2}+70 k+9\right) \lambda^{2}\right. \\
& \left.+\alpha^{2} \beta k\left(72 k^{2} \beta-14 k d+26 k \beta-5 d\right) \lambda-2 \alpha^{3} \beta k(3 k \beta-d)(4 k \beta-d)\right), \\
h_{6}= & \frac{\lambda k^{6}}{d^{5}}\left(k \alpha \beta\left(14+237 k+480 k^{3}+738 k^{2}\right) \lambda^{3}\right. \\
& -\left(178 k^{2}+30 k+1+292 k^{3}+120 k^{4}\right) \lambda^{4} \\
& -k \alpha^{2} \beta\left(720 k^{3} \beta+600 k^{2} \beta-94 k^{2} d+71 k \beta-79 k d-9 d\right) \lambda^{2} \\
& +k \alpha^{3} \beta\left(5 d^{2}+480 k^{3} \beta^{2}-188 k^{2} \beta d+16 k d^{2}-59 k \beta d\right) \lambda \\
& \left.-2 k \alpha^{4} \beta(5 k \beta-d)(3 k \beta-d)(4 k \beta-d)\right),
\end{aligned}
$$

where $k=\frac{\beta+n}{d}$.

Then, we get an approximation to $H$ as follows

$$
\begin{aligned}
H= & \frac{-\lambda k^{2}}{d} x_{2}^{2}+\frac{\lambda k^{3}}{d^{2}}(-2 k \alpha \beta+2 \lambda k+\lambda) x_{2}^{3}+\frac{-k^{4} \lambda}{d^{3}}\left(\left(7 k+1+6 k^{2}\right) \lambda^{2}\right. \\
& \left.-k \alpha \beta(5+12 k) \lambda+2 k \alpha^{2} \beta(3 k \beta-2 d)\right) x_{2}^{4}+\frac{\lambda k^{5}}{d^{4}}\left(\left(24 k^{3}+44 k^{2}+16 k+1\right) \lambda^{3}\right. \\
& -k \alpha \beta\left(72 k^{2}+70 k+9\right) \lambda^{2}+\alpha^{2} \beta k\left(72 k^{2} \beta-14 k d+26 k \beta-5 d\right) \lambda \\
& \left.-2 \alpha^{3} \beta k(3 k \beta-d)(4 k \beta-d)\right) x_{2}^{5}+\frac{\lambda k^{6}}{d^{5}}\left(k \alpha \beta\left(14+237 k+480 k^{3}+738 k^{2}\right) \lambda^{3}\right. \\
& -\left(178 k^{2}+30 k+1+292 k^{3}+120 k^{4}\right) \lambda^{4} \\
& -k \alpha^{2} \beta\left(720 k^{3} \beta+600 k^{2} \beta-94 k^{2} d+71 k \beta-79 k d-9 d\right) \lambda^{2} \\
& +k \alpha^{3} \beta\left(5 d^{2}+480 k^{3} \beta^{2}-188 k^{2} \beta d+16 k d^{2}-59 k \beta d\right) \lambda \\
& \left.-2 k \alpha^{4} \beta(5 k \beta-d)(3 k \beta-d)(4 k \beta-d)\right) x_{2}^{6}+\cdots .
\end{aligned}
$$

Substituting (8) into the second equation of system (9) leads to the vector field reduced to the center manifold

$$
\begin{aligned}
\frac{d z_{2}}{d t}= & k^{2}(\lambda-\alpha \beta) x_{2}^{2}+\frac{k^{3}\left(\alpha^{2} \beta d-\lambda^{2}\right)}{d} x_{2}^{3}-\frac{k^{4}\left(\alpha^{3} \beta d^{2}-2 \lambda^{2} k \alpha \beta-2 \lambda^{3} k-\lambda^{3}\right)}{d^{2}} x_{2}^{4} \\
& -\frac{k^{5}}{d^{3}}\left((k+1)(6 k+1) \lambda^{4}-\alpha k \beta(12 k+5) \lambda^{3}\right. \\
& \left.+2 \alpha^{2} k \beta(3 k \beta-d) \lambda^{2}-\alpha^{4} \beta d^{3}\right) x_{2}^{5} \\
& +\frac{k^{6}}{d^{4}}\left(\left(24 k^{3}+44 k^{3}+16 k+1\right) \lambda^{5}-k \alpha \beta\left(27 k^{2}+70 k+9\right) \lambda^{4}\right.
\end{aligned}
$$




$$
\begin{aligned}
& +\alpha^{3} k \beta\left(26 k \beta+72 k^{2} \beta-14 k d-5 d\right) \lambda^{3} \\
& \left.-2 \alpha^{3} k \beta(3 k \beta-d)(4 k \beta-d) \lambda^{2}-\alpha^{5} \beta d^{4}\right) x_{2}^{6}+\cdots .
\end{aligned}
$$

Then, by the center manifold theorem, we have the following results about the non-hyperbolic equilibrium $E_{0}$.

Theorem 6 Under the condition of $R_{0}=1$, the disease free equilibrium $E_{0}$ is asymptotically stable if $\lambda<\alpha \beta$, and unstable if $\lambda>\alpha \beta$.

Remark 7 If $R_{1} \geq 1$, we have $\lambda<\alpha \beta$. Then, if $R_{1} \geq 1$ and $R_{0}=1, E_{0}$ is aseptically stable.

By the Lyapunov's second method, we have the following result with respect to the globally asymptotically stability of the disease free equilibrium $E_{0}$.

Theorem 8 If $R_{0}<1-\frac{\beta \alpha A}{d(d+\alpha A)(\beta+n)}, E_{0}$ is globally asymptotically stable.

Proof Let $V_{2}=\frac{I^{2}}{2}$, then the total differential of $V$ along the flow of (5) is

$$
\left.\frac{d V}{d t}\right|_{(5)}=\left(\lambda S-n-\frac{\beta}{1+\alpha I}\right) I^{2} .
$$

Note that $\mathbb{O}$ is the positively invariant set of system (5), we restrict $(S, I) \in \mathbb{O}$, and which leads to $\lambda S \leq \frac{\lambda A}{d}$. Then, we have

$$
\left.\frac{d V}{d t}\right|_{(5)} \leq\left(d(\beta+n)\left(R_{0}-1\right)+\frac{\beta \alpha A}{d+\alpha A}\right) I^{2} .
$$

By the Lyapunov-Lasalle theorem [5], $E_{0}$ is globally stable if $R_{0}<1-$ $\frac{\beta \alpha A}{d(d+\alpha A)(\beta+n)}$.

Next, we study the stability of the endemic equilibrium $E_{2}$ under the conditions:

$$
R_{0}=1, \quad R_{1}>1 .
$$

Clearly, (13) leads to

$$
\lambda<\alpha d, \quad A>\frac{n d}{\lambda}, \quad \beta=\frac{\lambda A}{d}-n .
$$

The Jacobian matrix of system (5) at $E_{2}$ has the form

$$
M_{E_{2}}:=\left(\begin{array}{cc}
-d-\lambda I_{2}^{*} & -\lambda S_{2}^{*} \\
\lambda I_{2}^{*} & \frac{\alpha \beta I_{2}^{*}}{\left(1+\alpha I_{2}^{*}\right)}
\end{array}\right) .
$$

It is easy to obtain the characteristic equation of $M_{E_{2}}$ is

$$
a_{1} \theta^{2}+a_{2} \theta+a_{3}=0,
$$


where $\theta$ is a complex number and

$$
\begin{aligned}
& a_{1}=\alpha n d(\lambda A-n d)(\lambda-\alpha d)^{2}, \\
& a_{2}=b_{1} A^{2}+b_{2} A+b_{3}, \\
& a_{3}=n(\alpha d-\lambda)\left(-\alpha d \lambda A+\alpha d^{2} n+\lambda^{2} A\right)^{2}, \\
& b_{1}=-\lambda^{2}(\lambda-\alpha d)^{3}, \\
& b_{2}=\lambda n d(\lambda-\alpha d)\left((\lambda-\alpha d)^{2}+\lambda n \alpha\right), \\
& b_{3}=\alpha^{2} n^{3} \lambda d^{3}>0 .
\end{aligned}
$$

By using (13-14), we get

$$
a_{1}>0, \quad a_{3}>0, \quad b_{1}>0, \quad b_{2}<0 .
$$

Then, we obtain

Theorem $9 E_{2}$ is asymptotically stable if $a_{2}>0$ and unstable if $a_{2}<0$.

Let $\Delta_{1}=b_{2}^{2}-4 b_{1} b_{3}$. It is easy to see that equation $a_{2}=0$ has positive root $A$ if and only if $\Delta_{1} \geq 0$. Without lose of generality, we suppose $\Delta_{1}>0$. Then, $a_{2}=0$ has two distinct solutions: $A_{1}=\frac{-b_{2}-\sqrt{\Delta_{1}}}{2 b_{1}}, A_{2}=\frac{-b_{2}+\sqrt{\Delta_{1}}}{2 b_{1}}$. Obviously, (15) has a simple pair of pure imaginary roots $\lambda_{1,2}= \pm \omega i$ if $A=A_{j}$, where $\omega=\sqrt{\frac{a_{3}}{a_{1}}}=$ $\sqrt{\frac{\left(-\alpha d \lambda A+\alpha d^{2} n+\lambda^{2} A\right)^{2}}{\alpha d(\lambda A-n d)(\alpha d-\lambda)}}, i$ is the imaginary unit, $j=1,2$. It is easy to obtain that $\left.\frac{d \Re \mathfrak{e} \lambda_{1,2}}{d A}\right|_{A=A_{j}}=-\left.\frac{a_{2}}{2 a_{1}}\right|_{A=A_{j}}$, where $a_{2}$ denotes the derivative of $a_{2}$ with respect to $A$. Then, we have $\left.\frac{d \mathfrak{R} \lambda_{1,2}}{d A}\right|_{A=A_{1}}>0$ and $\left.\frac{d \mathfrak{R} \mathfrak{e} \lambda_{1,2}}{d A}\right|_{A=A_{2}}<0$. By the Hopf bifurcation theorem [4, 16], a Hopf bifurcation occurs at the endemic equilibrium $E_{2}$ when $A$ passes through each critical value $A_{j}, j=1,2$.

Next, under the condition of $a_{2}=0$, we verify the stability of the bifurcated periodic orbits which occurs near the positive equilibrium. For the end, we need to compute the index number in the Hopf bifurcation theorem. Let $x_{1}=S-S^{*}$ and $x_{2}=I-I_{2}^{*}$, system (5) can be transformed into

$$
\left\{\begin{array}{l}
\frac{d x_{1}}{d t}=-\left(d+\lambda I_{2}^{*}\right) x_{1}-\lambda S_{2}^{*} x_{2}-\lambda x_{1} x_{2}, \\
\frac{d x_{2}}{d t}=\lambda I_{2}^{*} x_{1}+\frac{\alpha \beta I_{2}^{*}}{\left(1+\alpha I_{2}^{*}\right)^{2}} x_{2}+\lambda x_{1} x_{2}-\left(\frac{\beta\left(x_{2}+I_{2}^{*}\right)}{1+\alpha\left(x_{2}+I_{2}^{*}\right)}-\frac{\beta I_{2}^{*}}{1+\alpha I_{2}^{*}}-\frac{\beta}{\left(1+\alpha I_{2}^{*}\right)} x_{2}\right) .
\end{array}\right.
$$

Obviously, $M_{E_{2}}$ can be re-expressed as

$$
M_{E_{2}}=\left(\begin{array}{cc}
\frac{\lambda A_{j} n_{1}}{n_{4}} & \frac{n_{4}}{n_{1}} \\
-\frac{n_{3}}{n_{4}} & -\frac{\lambda n n_{3}}{n_{1}^{2} n_{2}}
\end{array}\right)
$$

where $n_{1}=\lambda-\alpha d<0, n_{2}=\lambda A_{j}-n d>0, n_{3}=\alpha d^{2} n+\lambda^{2} A_{j}-\alpha d \lambda A_{j}<0$, $n_{4}=\alpha d n>0, j=1,2$. 
Let $\lambda_{1,2}= \pm \omega i$ be the pure imaginary roots of $M_{E_{2}}$ and

$$
\left(\begin{array}{l}
x_{1}(t) \\
z_{2}(t)
\end{array}\right)=T\left(\begin{array}{l}
y_{1}(t) \\
y_{2}(t)
\end{array}\right) \quad \text { with } T=\left(\begin{array}{cc}
\frac{n_{4}}{n_{1}} & 0 \\
-\frac{\lambda A n_{1}}{n_{4}} & \omega
\end{array}\right),
$$

then system (16) becomes

$$
\left\{\begin{array}{l}
\frac{d y_{1}}{d t}=\omega y_{2}+f_{1}\left(y_{1}, y_{2}\right) \\
\frac{d y_{2}}{d t}=-\omega y_{1}+f_{2}\left(y_{1}, y_{2}\right)
\end{array}\right.
$$

where

$$
\begin{aligned}
f_{1}\left(y_{1}, y_{2}\right)= & -\frac{\lambda n_{4}}{n_{1}}\left(-\frac{\lambda A n_{1}}{n_{4}} y_{1}+\omega y_{2}\right) y_{2}, \\
f_{2}\left(y_{1}, y_{2}\right)= & \frac{\lambda n_{4}}{n_{1}}\left(-\frac{\lambda A n_{1}}{n_{4}} y_{1}+\omega y_{2}\right) y_{2}-\left(\frac{\beta\left(\left(-\frac{\lambda A_{j} n_{1}}{n_{4}} y_{1}+\omega y_{2}\right)+I_{2}^{*}\right)}{1+\alpha\left(\left(-\frac{\lambda A_{j} n_{1}}{n_{4}} y_{1}+\omega y_{2}\right)+I_{2}^{*}\right)}\right. \\
& \left.-\frac{\beta I_{2}^{*}}{1+\alpha I_{2}^{*}}-\frac{\beta}{\left(1+\alpha I_{2}^{*}\right)^{2}}\left(-\frac{\lambda A_{j} n_{1}}{n_{4}} y_{1}+\omega y_{2}\right)\right) .
\end{aligned}
$$

For convenience, we rewrite $f_{1}, f_{2}$ as $f^{1}, f^{2}$, respectively. Therefore, we can compute the index $K$ in [4] as follows

$$
\begin{aligned}
K= & \frac{1}{16}\left[f_{x_{1} x_{1} x_{1}}^{1}+f_{x_{1} x_{2} x_{2}}^{1}+f_{x_{1} x_{1} x_{2}}^{2}+f_{x_{2} x_{2} x_{2}}^{2}\right]+\frac{1}{16 \omega}\left[f_{x_{1} x_{2}}^{1}\left(f_{x_{1} x_{1}}^{1}+f_{x_{2} x_{2}}^{1}\right)\right. \\
& \left.-f_{x_{1} x_{2}}^{2}\left(f_{x_{1} x_{1}}^{2}+f_{x_{2} x_{2}}^{2}\right)-f_{x_{1} x_{1}}^{1} f_{x_{1} x_{1}}^{2}+f_{x_{2} x_{2}}^{1} f_{x_{2} x_{2}}^{2}\right] \\
= & -\frac{3 \beta \alpha^{2} \omega\left(\lambda^{2} A_{j}^{2} n_{1}^{2}+\omega^{2} n_{4}^{2}\right)}{8\left(1+\alpha I_{2}^{*}\right)^{4} n_{4}^{2}}-\frac{1}{16 \omega}\left[-\frac{2 \lambda^{3} \omega A_{j} n_{1} n_{4}}{n_{2}^{2}}\right. \\
& +\frac{2 \lambda^{2} A_{j} n_{1}}{n_{2}}\left(\frac{2 \beta \lambda^{2} A_{j}^{2} n_{1}^{2} \alpha}{n_{4}^{2}\left(1+\alpha I_{2}^{*}\right)^{2}}-\frac{2 \lambda^{2} A_{j} n_{1}}{n_{2}}-\frac{2 \beta I_{2}^{*} \alpha^{2} \lambda^{2} A_{j}^{2} n_{1}^{2}}{\left(1+\alpha I_{2}^{*}\right)^{3} m_{4}^{2}}\right) \\
& +\left(\frac{\lambda n_{4} \omega}{n_{2}}-\frac{2 \beta \lambda A_{j} n_{1} \alpha \omega}{n_{4}\left(1+\alpha I_{2}^{*}\right)}+\frac{2 \beta I_{2}^{*} \alpha^{2} \lambda A_{j} n_{1} \omega}{\left(1+\alpha I_{2}^{*}\right)^{3} n_{4}}\right) \\
& \times\left(\frac{2 \beta \lambda^{2} A_{j}^{2} n_{1}^{2} \alpha}{n_{4}^{2}\left(1+\alpha I_{2}^{*}\right)^{2}}-\frac{2 \lambda^{2} A_{j} n_{1}}{n_{2}}-\frac{2 \beta I_{2}^{*} \alpha^{2} \lambda^{2} A_{j}^{2} n_{1}^{2}}{\left(1+\alpha I_{2}^{*}\right)^{3} n_{4}^{2}}\right. \\
& +\frac{2 \beta \omega^{2} \alpha}{\left.\left.\left(1+\alpha I_{2}^{*}\right)^{2}-\frac{2 \beta I_{2}^{*} \alpha^{2} \omega^{2}}{\left(1+\alpha I_{2}^{*}\right)^{3}}\right)\right] .}
\end{aligned}
$$

By using the Hopf bifurcation theorem, we obtain the following results about the periodic solutions bifurcated at $E_{2}$. 
Theorem 10 If $K<0$, the periodic solution is stable, while if $K>0$, the periodic solution is unstable. The case $K<0$ is referred to as a supercritical bifurcation, and the case $K>0$ is referred to as a subcritical bifurcation.

In the following, the dynamic property of $E_{4}$ is focused on under the conditions:

$$
R_{0}<1, \quad R_{1}>1, \quad \Delta=0 .
$$

The Jacobian matrix of system (5) at $E_{4}$ is

$$
M_{E_{4}}:=\left(\begin{array}{cc}
-d-\lambda I_{4}^{*} & -\lambda S_{4}^{*} \\
\lambda I_{4}^{*} & \frac{\alpha \beta I_{4}^{*}}{\left(1+\alpha I_{4}^{*}\right)^{2}}
\end{array}\right) .
$$

By using the relationship $\left(I_{4}^{*}\right)^{2}=\frac{(n(\alpha d+\lambda)+\lambda \beta-\lambda A \alpha) I_{4}^{*}+(\beta+n) d-\lambda A}{\lambda n \alpha}$, we get the characteristic equation of $M_{E_{4}}$ is as follows

$$
\theta^{2}+p \theta+q=0
$$

where

$$
\begin{aligned}
p= & -\frac{1}{\left(1+\alpha I_{4}^{*}\right)^{2} n^{2}}\left[\left(-\lambda n A \alpha-\lambda \beta^{2}+2 \lambda \beta A \alpha+A \alpha^{2} n d-\lambda A^{2} \alpha^{2}+n^{2} \alpha \beta\right) I_{4}^{*}\right. \\
& \left.+\left(-\lambda A^{2} \alpha+\lambda \beta A-\beta^{2} d+d \alpha A n-\lambda A n+A \alpha d \beta\right)\right], \\
q= & \frac{I_{4}^{*}}{\left(1+\alpha I_{4}^{*}\right)^{2}}\left[\left(\alpha(n(d \alpha+\lambda)+\lambda \beta-\lambda A \alpha)-\alpha \beta \lambda+\lambda \alpha^{2}+2 \lambda n d\right) I_{4}^{*}\right. \\
& +\alpha((\beta+n) d-\lambda A)+\lambda(n+\alpha)-\alpha \beta d]
\end{aligned}
$$

and $\theta$ is a complex number.

Noticing $R_{1}>1$, we have $A>\frac{n d \alpha+(\beta+n) \lambda}{\alpha \lambda}$. For convenience, let $A=\frac{n d \alpha+(\beta+n) \lambda}{\alpha \lambda}$ $+\epsilon$, where $\epsilon>0$. By means of $\Delta=0$, we obtain

$$
\begin{aligned}
& p=\frac{(\alpha \epsilon+2 n)^{3}(\alpha \epsilon+2(\beta+n)) d-2 \epsilon \alpha \beta n\left((\alpha \epsilon+2 n)^{2}+4 \beta n\right)}{4 n^{2}\left(1+\alpha I_{4}^{*}\right)^{2}\left(\alpha^{2} \epsilon+4 \beta n+4 n^{2}+4 n \alpha \epsilon\right)}, \\
& q=\frac{2 \alpha \beta d I_{4}^{*}(\alpha-\beta)(d \epsilon+2 n)}{\left(1+\alpha I_{4}^{*}\right)^{2}\left(4 \beta n+(\alpha \epsilon+2 n)^{2}+4 n \beta\right)}, \\
& \lambda=\frac{4 n d \alpha \beta}{\alpha^{2} \epsilon^{2}+4 n \beta+4 n^{2}+4 n \epsilon \alpha}, \quad I_{4}^{*}=\frac{\epsilon}{2 n} .
\end{aligned}
$$

Let $d^{*}=\frac{2 \epsilon \alpha \beta n\left((\alpha \epsilon+2 n)^{2}+4 \beta n\right)}{(\alpha \epsilon+2 n)^{3}(\alpha \epsilon+2(\beta+n))}$. Then, under the condition of (17), we arrive at 


\section{Theorem 11}

(1) If $\alpha>\beta$ and $d>d^{*}, E_{4}$ is an asymptotically stable node or focus.

(2) If $\alpha>\beta$ and $d<d^{*}, E_{4}$ is an unstable node or focus.

(3) If $\alpha=\beta$, or $d=d^{*}, \alpha>\beta, E_{4}$ is a non-hyperbolic equilibrium.

(4) If $\alpha<\beta, E_{4}$ is a saddle.

Obviously, (18) has a negative root $-p$ except 0 if

$$
\alpha=\beta, \quad d>d^{*} .
$$

In the sequel, we discuss the stability of $E_{4}$ under the assumptions of (20). For the purpose, we first transfer $E_{4}$ to the origin by $x_{1}=S-S_{4}^{*}, x_{2}=I-I_{4}^{*}$. Therefore, we get

$$
\left\{\begin{array}{l}
\frac{d x_{1}}{d t}=-\frac{d(2(\beta+n)+\epsilon \beta)(2 n+\beta \epsilon)}{(\beta \epsilon+2 n)^{2}+4 n \epsilon \beta} x_{1}-\frac{n(2(\beta+n)+\beta \epsilon)}{2 n+\beta \epsilon} x_{2}+f_{1}\left(x_{1}, x_{2}\right), \\
\frac{d x_{2}}{d t}=\frac{2 d \beta^{2} \epsilon}{(\beta \epsilon+2 n)^{2}+4 n \beta} x_{1}+\frac{2 \beta \epsilon n}{(2 n+\epsilon \beta)^{2}} x_{2}+f_{2}\left(x_{1}, x_{2}\right),
\end{array}\right.
$$

where $f_{1}=\bar{a}_{1} x_{1} x_{2}, f_{2}=-\bar{a}_{1} x_{1} x_{2}+b_{2} x_{2}^{2}+b_{3} x_{2}^{3}+b_{4} x_{2}^{4}+b_{5} x_{2}^{5}+O\left(x_{2}^{6}\right), \bar{a}_{1}=$ $-\frac{4 n d \beta^{2}}{(\beta \epsilon+2 n)^{2}+4 n \beta}, \bar{k}=\frac{2 \beta n}{2 n+\beta \epsilon}, b_{j}=(-1)^{j} \frac{\bar{k}^{j}}{\beta}, j=2,3,4,5$.

Secondly, we define

$$
\left(\begin{array}{l}
x_{1} \\
x_{2}
\end{array}\right)=S\left(\begin{array}{l}
y_{1} \\
y_{2}
\end{array}\right), \quad S=\left(\begin{array}{ll}
s_{11} & s_{12} \\
s_{21} & s_{22}
\end{array}\right),
$$

where $s_{11}=-n\left((\beta \epsilon+2 n)^{2}+4 \beta n\right), s_{12}=-\left((\beta \epsilon+2 n)^{2}+2 \beta(\beta \epsilon+2 n)\right), s_{21}=$ $d(2 n+\beta \epsilon), s_{22}=2 \epsilon \beta^{2}$.

It isn't difficult to get the inverse of $S$ as follows

$$
S^{-1}=\left(\begin{array}{ll}
t_{11} & t_{12} \\
t_{21} & t_{22}
\end{array}\right)
$$

where $\quad t_{11}=\frac{2 \epsilon \beta^{2}}{\operatorname{det}(S)}, \quad t_{12}=\frac{(2 n+\epsilon \beta)(2(n+\beta)+\epsilon \beta)}{\operatorname{det}(S)}, \quad t_{21}=\frac{-d(2 n+\epsilon \beta)^{2}}{\operatorname{det}(S)}, \quad t_{22}=$ $\frac{-n\left(4 n^{2}+4 n \epsilon \beta+\epsilon^{2} \beta^{2}+4 n \beta\right)}{\operatorname{det}(S)}$.

Corresponding, system (21) becomes

$$
\left(\begin{array}{c}
\frac{d y_{1}}{d t} \\
\frac{d y_{2}}{d t}
\end{array}\right)=\left(\begin{array}{cc}
0 & 0 \\
0 & -p
\end{array}\right)\left(\begin{array}{l}
y_{1} \\
y_{2}
\end{array}\right)+\left(\begin{array}{l}
F_{1}\left(y_{1}, y_{2}\right) \\
F_{2}\left(y_{1}, y_{2}\right)
\end{array}\right)
$$

where

$$
\begin{aligned}
F_{1}= & \bar{a}_{1} t_{11}\left(s_{11} y_{1}+s_{12} y_{2}\right)\left(s_{21} y_{1}+s_{22} y_{2}\right)+t_{12}\left(-\bar{a}_{1}\left(s_{21} y_{1}+s_{22} y_{2}\right)^{2}\right. \\
& +b_{2}\left(s_{21} y_{1}+s_{22} y_{2}\right)^{2}+b_{3}\left(s_{21} y_{1}+s_{22} y_{2}\right)^{3}+b_{4}\left(s_{21} y_{1}+s_{22} y_{2}\right)^{4} \\
& \left.+b_{4}\left(s_{21} y_{1}+s_{22} y_{2}\right)^{5}\right)+O\left(\|y\|^{6}\right)
\end{aligned}
$$




$$
\begin{aligned}
F_{2}= & \bar{a}_{1} t_{21}\left(s_{11} y_{1}+s_{12} y_{2}\right)\left(s_{21} y_{1}+s_{22} y_{2}\right)+t_{22}\left(-\bar{a}_{1}\left(s_{21} y_{1}+s_{22} y_{2}\right)^{2}\right. \\
& +b_{2}\left(s_{21} y_{1}+s_{22} y_{2}\right)^{2}+b_{3}\left(s_{21} y_{1}+s_{22} y_{2}\right)^{3}+b_{4}\left(s_{21} y_{1}+s_{22} y_{2}\right)^{4} \\
& \left.+b_{4}\left(s_{21} y_{1}+s_{22} y_{2}\right)^{5}\right)+O\left(\|y\|^{6}\right) \\
y= & \left(y_{1}, y_{2}\right)^{T} \in \mathbb{R}^{2} .
\end{aligned}
$$

By the existence theorem in the center manifold theory, there exists a center manifold for system (24), which can be expressed locally as follows

$$
W^{c}(0)=\left\{\left(y_{1}, y_{2}\right) \in \mathbb{R}^{2}\left|y_{2}=\bar{H}\left(y_{1}\right),\left\|y_{1}\right\|<\delta, \bar{H}(0)=0, D \bar{H}(0)=0,\right| \delta>0\right\}
$$

where $\delta$ is sufficiently small and $D \bar{H}$ is the derivative of $\bar{H}$ with respect to $y_{1}$.

Obviously, the first task is to compute the center manifold $W^{c}(0)$. For the purpose, let $\bar{H}\left(y_{1}\right)$ be

$$
y_{2}=\bar{H}\left(y_{1}\right)=\bar{h}_{2} y_{1}^{2}+\bar{h}_{3} y_{1}^{3}+\bar{h}_{4} y_{1}^{4}+\cdots .
$$

By the invariance of $W^{c}(0)$ under the dynamics of $(24)$, the center manifold satisfies

$$
D h \cdot F_{1}\left(y_{1}, \bar{H}\left(y_{1}\right)\right)+p \bar{H}\left(y_{1}\right)-F_{2}\left(y_{1}, \bar{H}\left(y_{1}\right)\right)=0 \text {. }
$$

Balance equation (24) with respect to $x_{1}$ yields

$$
\begin{aligned}
& \bar{h}_{2}=\frac{s_{21}}{\operatorname{det}(S)}\left(t_{22} b_{2} s_{21}-t_{22} \bar{a}_{1} s_{21}+t_{21} \bar{a}_{1} s_{11}\right), \\
& \bar{h}_{3}=\frac{s_{21}}{p^{2}}\left(c_{1} s_{21}^{3}+c_{2} s_{21}^{2}+c_{3} s_{21}+c_{4}\right)
\end{aligned}
$$

with

$$
\begin{aligned}
c_{1}= & -2 t_{22} t_{11}\left(\bar{a}_{1}-b_{2}\right)^{2}, c_{4}=-s_{22} s_{21}^{2} \bar{a}_{1}^{2} t_{21}^{2}, \\
c_{3}= & -t_{21} \bar{a}_{1} s_{11}\left(2 \bar{a}_{1} t_{11} s_{11}-\bar{a}_{1} s_{21} t_{21}+3 \bar{a}_{1} s_{22} t_{22}-3 s_{22} t_{22} b_{2}\right), \\
c_{2}= & 2\left(\bar{a}_{1}-b_{2}\right)^{2} s_{22} t_{22}^{2}+t_{22}\left(b_{3} p-2 b_{2} \bar{a}_{1} s_{11} t_{11}-\bar{a}_{1}^{2} s_{12} t_{12}+2 \bar{a}_{1}^{2} s_{11} t_{11}+b_{2} s_{12} s_{21}\right) \\
& +2 \bar{a}_{1} t_{21} t_{12}\left(\bar{a}_{1}-b_{2}\right) .
\end{aligned}
$$

Substituting (23) into the first equation of system (24) leads to

$$
\frac{d y_{1}}{d t}=d_{2} y_{1}^{2}+d_{3} y_{1}^{3}+O\left(y_{1}^{4}\right)
$$

with

$$
\begin{aligned}
d_{2}= & \frac{s_{21}}{\operatorname{det}(S)}\left(s_{21}\left(-\left(\operatorname{det}(S) t_{12}\right) \bar{a}_{1} s_{21}+\left(\operatorname{det}(S) t_{12}\right) b_{2} s_{21}+\left(\operatorname{det}(S) t_{11}\right) \bar{a}_{1} s_{11}\right)\right), \\
d_{3}= & \frac{s_{21}}{p}\left(\left(-2 t_{12} b_{2}^{2} t_{22}+4 t_{12} b_{2} t_{22} \bar{a}_{1}-2 t_{12} \bar{a}_{1}^{2} t_{22}\right) s_{21}^{3}\right. \\
& +\left(-4 t_{22}^{2} \bar{a}_{1} s_{22} b_{2}-2 t_{11} \bar{a}_{1} s_{11} t_{22} b_{2}+2 t_{11} \bar{a}_{1}^{2} s_{11} t_{22}+t_{22} b_{3} p+t_{21} \bar{a}_{1} s_{12} t_{22} b_{2}\right.
\end{aligned}
$$




$$
\begin{aligned}
& \left.-t_{21} \bar{a}_{1}^{2} s_{12} t_{22}+2 t_{22}^{2} \bar{a}_{1}^{2} s_{22}+2 t_{22}^{2} b_{2}^{2} s_{22}-2 t_{12} b_{2} t_{21} \bar{a}_{1} s_{11}+2 t_{12} \bar{a}_{1}^{2} t_{21} s_{11}\right) s_{21}^{2} \\
& +\left(-2 t_{11} \bar{a}_{1}^{2} s_{11}^{2} t_{21}-3 t_{21} \bar{a}_{1}^{2} s_{11} s_{22} t_{22}+t_{21}^{2} \bar{a}_{1}^{2} s_{12} s_{11}\right. \\
& \left.\left.+3 t_{21} \bar{a}_{1} s_{11} s_{22} t_{22} b_{2}\right) s_{21}+s_{22} s_{11}^{2} \bar{a}_{1}^{2} t_{21}^{2}\right) .
\end{aligned}
$$

Noticing $\bar{a}_{1}<0, b_{2}>0, s_{21}>0, s_{11}<0, \operatorname{det}(S) t_{12}>0, H t_{11}>0$, we have $d_{2} \operatorname{det}(S)>0$. Then, by the center manifold theorem, we arrive at

Theorem 12 Under the conditions of (17) and (20), the endemic equilibrium $E_{4}$ is asymptotically stable if $\operatorname{det}(S)<0$, and unstable if $\operatorname{det}(S)>0$.

Suppose both $E_{1}$ and $E_{3}$ are hyperbolic equilibria. In the following, under the conditions of

$$
R_{0}<1, \quad R_{1}>1, \quad \Delta>0
$$

we investigate the stability of $E_{1}$ and $E_{3}$ by the technique presented in $[1,12]$ related to the Poincare index (see $[13,18]$ for the detail).

By Theorem 3.1, the disease free equilibrium $E_{0}$ is asymptotically stale if $R_{0}<1$. Suppose $\Omega$ is a small neighborhood of $E_{0}$, and which lies in the domain of attraction of $E_{0}$. Let $\mathbb{U}=\mathbb{O} \cup \bar{\Omega}$, where $\Omega$ is the closure of $\Omega$. Then $\mathbb{U}$ is a positively invariant set of system (5). Let $\mathfrak{L}$ be the boundary of $\mathbb{U}$. It follows from the Theorem 2.1 of [12] that the Poincare index of $\mathfrak{L}$ related to the vector field $F$ is 1 , i.e.

$$
a-s+r=1
$$

where $F$ represents the right hand sides of the equations in system (5), and $a, s$ and $r$ are the number of attractors (nodes), saddles and repellers (sources), respectively (see [13] for the detail). Since there are three hyperbolic equilibria in $\mathbb{U}$, we have

$$
a+s+r \leq 3 .
$$

Combining (26) with (27), we get $s \leq 1$.

If $s=0$, (26) leads to $r=0$, and further system (5) only have the disease free equilibrium $E_{0}$. Obviously, it is impossible if the condition (17) holds.

If $s=1$, (26) leads to $a+r=2$. Then, by using Theorem 3.1 and (27), we have that $a \geq 1$, and further have $r \leq 1$. Clearly, $a=2$ if $r=0$, while $a=1$ if $r=1$. The first case implies the disease free equilibrium $E_{0}$ and one of the two endemic equilibria are asymptotically stable, and the other endemic equilibrium is unstable, i.e., system (5) exhibits bi-stability. The second case means that both of the two endemic equilibria are unstable. Summarizing the above discussion yields

Theorem 13 Under the conditions of (25), either one of the two endemic equilibria (i.e. $\left.E_{1}, E_{3}\right)$ is asymptotically stable and the other endemic equilibrium is unstable or both of them are unstable. 
Finally, we discuss the stability of the endemic equilibrium $E_{1}$ under the condition of $R_{0}>1$. Clearly, the Jacobian matrix of system (5) at $E_{1}$ has the form

$$
M_{E_{1}}:=\left(\begin{array}{cc}
-d-\lambda I_{1}^{*} & -\frac{n+n \alpha I_{1}^{*}+\beta}{1+\alpha I_{1}^{*}} \\
\lambda I_{1}^{*} & \frac{\alpha \beta I_{1}^{*}}{\left(1+\alpha I_{1}^{*}\right)^{2}}
\end{array}\right) .
$$

Let $p=d+\lambda I_{1}^{*}-\frac{\alpha \beta I_{1}^{*}}{\left(1+\alpha I_{1}^{*}\right)^{2}}, q=\frac{\lambda^{2} A}{d+\lambda I_{1}^{*}} q_{1}\left(I_{1}^{*}\right)$, where $q_{1}\left(I_{1}^{*}\right)=1-\frac{\alpha \beta\left(d+\lambda I_{1}^{*}\right)^{2}}{\lambda^{2} A\left(1+\alpha I_{1}^{*}\right)^{2}}$. Clearly, $q_{1}$ is decrease with respect to $I_{1}^{*}$ when $\lambda>\alpha d$ and increase when $\lambda<\alpha d$. Note that $p \geq d-\frac{\beta}{4}+\lambda I_{1}^{*}$ and $q_{1}(0)=1-\frac{\alpha \beta d^{2}}{\lambda^{2} A}$, then, under the conditions of $R_{0}>1$ and $d>\frac{\beta}{4}$, we have

Theorem 14 If one of the following is satisfied: (1) $I \lambda \geq \alpha d$ and $A \leq \frac{\beta}{\alpha}$, (2) $\lambda<\alpha d$ and $A \leq \frac{(\beta+n)^{2}}{\alpha \beta}$, then $E_{1}$ is asymptotically stable.

Proof (1) By using $R_{0}>1$, we get $q_{1}(0)>\frac{\lambda d(\beta+n)-\alpha \beta d^{2}}{\lambda A}$. If $\lambda \geq \alpha d$, then $q_{1}(0) \geq$ $\frac{n d^{2} \alpha}{\lambda^{2} A}>0$. Then $q>0$ if $\lim _{I_{1}^{*} \rightarrow \infty} q_{1}\left(I_{1}^{*}\right)=1-\frac{\beta}{A \alpha}>0$. So, $E_{1}$ is asymptotically stable if $\lambda \geq \alpha d$ and $A \leq \frac{\beta}{\alpha}$.

(2) Obviously, $R_{0}>1$ leads to $d<\frac{\lambda A}{\beta+n}$. Then, we have $q_{1}(0)$ yields $q_{1}(0)>$ $1-\frac{A \alpha \beta}{(\beta+n)^{2}}$ and further have $q>0$ if $A \leq \frac{(\beta+n)^{2}}{\alpha \beta}$.

By Bendixion-Poincaré theorem, it isn't difficult to obtain

Theorem 15 If $p>0$ and $q>0$, and further $\lambda<\frac{d^{3}}{\alpha A^{2}}$, the endemic equilibrium $E_{1}$ is globally asymptotically stable, while if $p<0$ and $q>0$ it is unstable and there at least exists one periodic orbit.

\section{Numerical simulation}

In this section, to understand our results more intuitively, some numerical simulations are carried out. Six figures are employed to exhibit the dynamic behaviors of system (5), such as the stability of the endemic equilibria, the existences of the periodic orbit and the bistable equilibria, respectively.

Firstly, let $R_{0}>1$. Then there exist a unstable disease free equilibrium $E_{0}$ and a unique endemic equilibrium $E_{1}$. Unlike the traditional epidemic model, the stability of the unique endemic equilibrium $E_{1}$ is not invariant. It can be asymptotically stable and also can be unstable. In Fig. 1, $E_{1}$ is asymptotically stable. However, in Fig. 2, it is unstable and at least one periodic orbit occurs.

Secondly, let $R_{0}=1$. Then there exists a unique endemic equilibrium $E_{2}$ if $R_{1}>1$, and its stability depends on the parameters which is sketched in Figs. 3-4, respectively. 
Fig. $1 R_{0}>1, A=7.65$, $d=0.5, \lambda=0.5, n=0.75$, $\beta=2, \alpha=0.5 . E_{1}$ is asymptotically stable

Fig. $2 R_{0}>1, A=76.5$, $d=0.5, \lambda=0.5, n=7.5$, $\beta=20, \alpha=0.5 . E_{1}$ is unstable, and there at least exists a periodical orbit in the first quadrant of $(S, I)$ plane
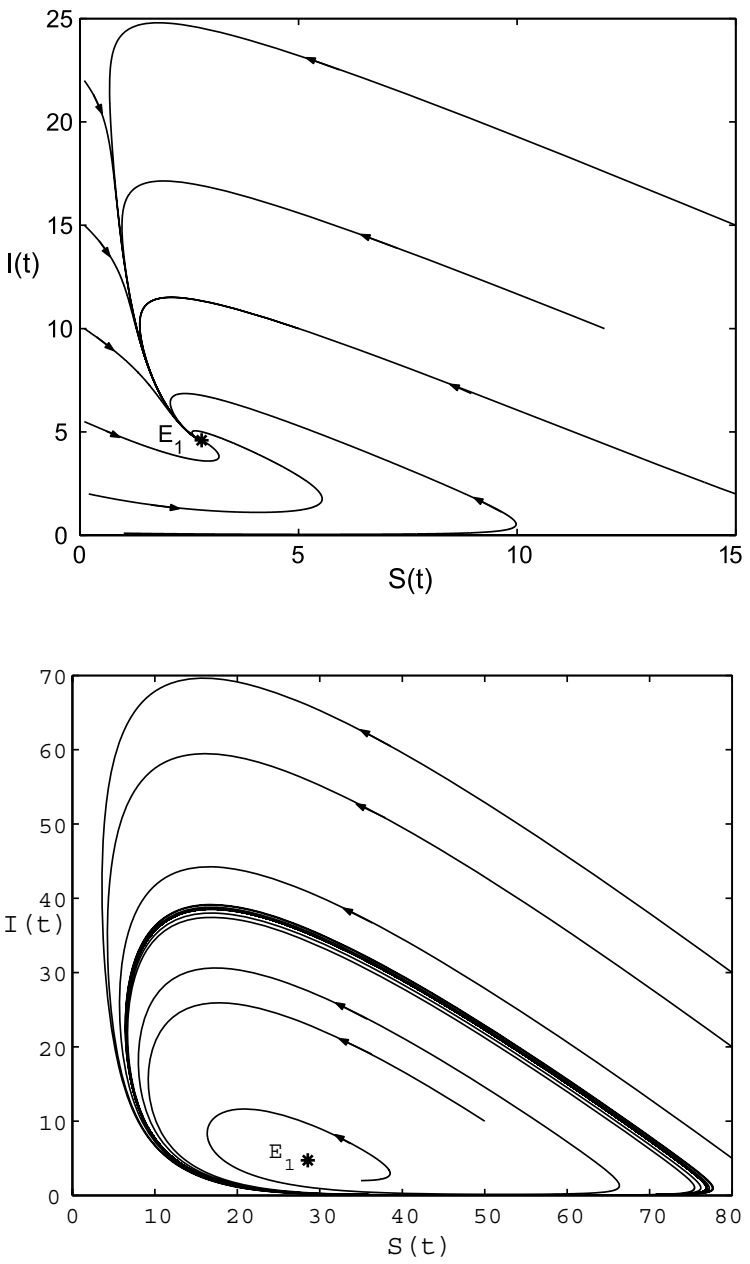

Fig. $3 R_{0}=1, R_{1}>1$, $A=523.6304348, d=0.5$, $\lambda=0.02, n=11.1$, $\beta=9.8452174, \alpha=0.5 . E_{2}$ is unstable, and the trajectories around it approach to the disease free equilibrium $E_{0}$

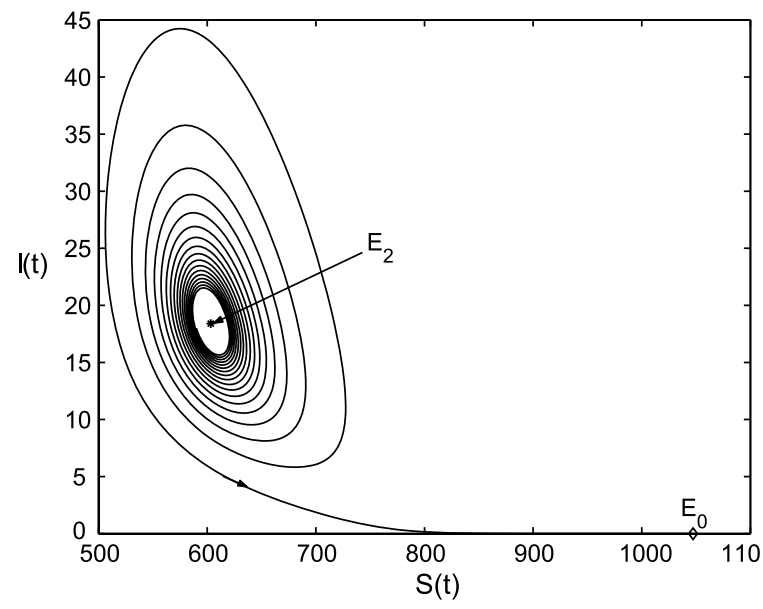


Fig. $4 R_{0}=1, R_{1}>1$, $A=1.17, d=0.005$, $\lambda=0.0001923076923$, $n=0.01, \beta=0.035, \alpha=0.05$. $E_{2}$ is asymptotically stable

Fig. $5 \quad R_{0}<1, R_{1}>1$, $A=14.65685425, d=1$, $\lambda=0.2, n=2, \beta=1, \alpha=0.35$, $\Delta=0$. Both the unique endemic equilibrium $E_{4}$ and the disease free equilibrium $E_{0}$ are asymptotically stable
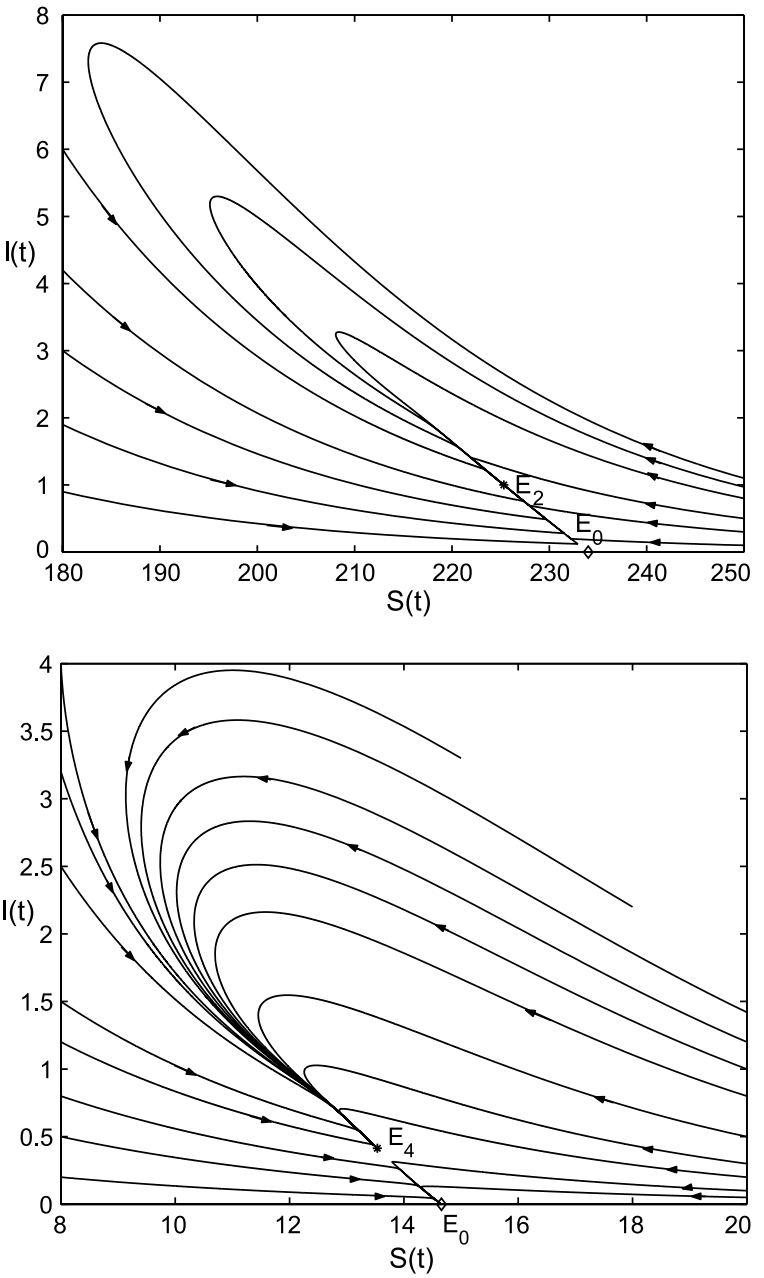

Fig. $6 R_{0}<1, R_{1}>1$, $A=392.5043354, d=15$, $\lambda=1.10624999, n=15.2$, $\beta=14.08052805, \alpha=0.2$, $\Delta>0$. Their exist two endemic equilibria $E_{1}$ and $E_{3}$ except the disease free equilibrium $E_{0}$. It is easy to see that $E_{1}$ and $E_{0}$ are bistable equilibria, while $E_{3}$ is a saddle

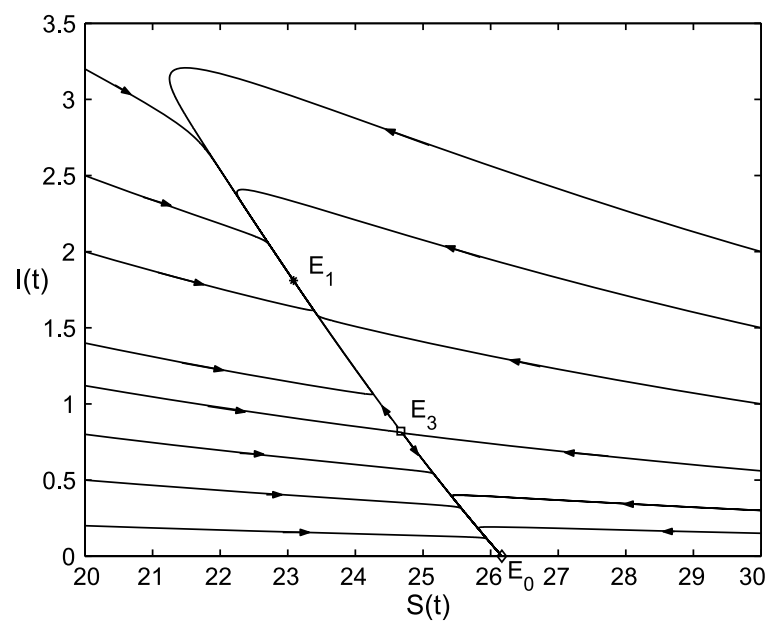


Finally, let $R_{0}<1$ and $R_{1}>1$. By Theorem 2.1, the existence of the endemic equilibrium depends on the sign of $\Delta$, i.e. system (5) has two endemic equilibria $E_{1}$, $E_{3}$ if $\Delta>0$ and a unique endemic equilibrium $E_{4}$ if $\Delta=0$. The dynamic behaviors of the endemic equilibria are showed in Figs. 5-6, respectively.

\section{Conclusions}

In the present paper, considering the perpetual immunity of the removed individuals and the saturated treatment rate, a SIR epidemic model is formulated, and which characterizes the effect of limited treatment capacity on the spread of epidemic disease. Unlike the traditional SIR model, multiple endemic equilibria co-exist in system (5), and it has bistable equilibria. Therefore, it is not always effective to reduce the basic reproduction number $R_{0}$ to the value less than the unity in the control and elimination of epidemic disease. In the same time, we have to control each sub-population to a given domain, and in which the disease free equilibrium $E_{0}$ is asymptotically stable.

By using the Hopf bifurcation theorem, Lyapunov-Lasalle theorem, Poincaré index, Poincaré-Bendixson, center manifold theorem and fixed point theorem, we analyze the existence and stability of endemic equilibria, the existence and direction of Hopf bifurcation. Finally, numerical simulation are presented, and which intuitively show the existence of bistable equilibria and the stability of each equilibrium.

\section{References}

1. Corbett, B.D., Moghadas, S.M., Gumel, A.B.: Subthreshold domain of bistable equilibria for a model of HIV epidemiology. Int. J. Math. Math. Sci. 2003, 3679-3698 (2003)

2. Cui, J., Mu, X., Wan, H.: Saturation recovery leads to multiple endemic equilibria and backward bifurcation. J. Theor. Biol. 254, 275-283 (2008)

3. Feng, Z., Thieme, H.R.: Recurrent outbreaks of childhood diseases revisited: the impact of isolation. Math. Biosci. 128, 93-130 (1995)

4. Gukenheimer, J., Holmes, P.: Nonlinear Oscillations, Dynamical Systems, and Bifurcations of Vector Fields. Springer, Berlin (1990)

5. Hale, J.K.: Ordinary Differential Equations, 2nd edn. Krieger, Melbourne (1980)

6. Hethcote, H.W.: The mathematics of infectious diseases. SIAM Rev. 42, 599-653 (2000)

7. Hyman, J.M., Li, J.: Modeling the effectiveness of isolation strategies in preventing STD epidemics. SIAM J. Appl. Math. 58, 912-925 (1998)

8. Khalil, H.K.: Nonlinear Systems, 3rd edn. Prentice-Hall, Englewood Cliffs (2002)

9. Kribs-Zaleta, C.M., Velasco-Hernsandez, J.X.: A simple vaccination model with multiple endemic states. Math. Biosci. 164, 183-201 (2000)

10. Matallana, L.G., Blanco, A.M., Bandoni, J.A.: Estimation of domains of attraction in epidemiological models with constant removal rates of infected individuals. J. Phys. Conf. Ser. 90, 1-7 (2007)

11. Miller, R.K., Michel, A.N.: Ordinary Differential Equations. Academic Press, San Diego (1982)

12. Moghadas, S.M.: Analysis of an epidemic model with bistable equilibria using the Poincaré index. Appl. Math. Comput. 149, 689-702 (2004)

13. Perko, L.: Differential Equations and Dynamical Systems. Springer, New York (1996)

14. Smith, H.L., Thieme, H.R.: Stable coexistence and bi-stability for competitive systems on ordered Banach spaces. J. Differ. Equ. 176, 195-222 (2001)

15. Wang, W., Ruan, S.: Bifurcations in an epidemic model with constant removal rate of the infectives. J. Math. Anal. Appl. 291, 775-793 (2004) 
16. Wiggins, S.: Introduction to Applied Nonlinear Dynamical Systems and Chaos. Springer, Berlin (1990)

17. Wu, L., Feng, Z.: Homoclinic bifurcation in an SIQR model for childhood diseases. J. Differ. Equ. 168, 150-167 (2000)

18. Zhang, Z.F., Ding, T.R., Huang, W.Z., et al.: Qualitative Theory of Differential Equations. Science Press, Beijing (1985) (in Chinese) 